\title{
Activation of aldehyde dehydrogenase 2 slows down the progression of atherosclerosis via attenuation of ER stress and apoptosis in smooth muscle cells
}

\author{
Mei-yan YANG ${ }^{1,2}$, Ya-bin WANG ${ }^{1}$, Bo HAN ${ }^{2}$, Bo YANG ${ }^{1}$, Yu-wei QIANG ${ }^{1}$, Yan ZHANG ${ }^{1}$, Zhao WANG ${ }^{1}$, Xu HUANG $^{1}$, Jie LIU ${ }^{1}$, Yun- \\ dai $\mathrm{CHEN}^{1}$, Jun $\mathrm{REN}^{3, *}$, Feng $\mathrm{CAO}^{1, *}$, Yong $\mathrm{XU}^{1, *}$ \\ ${ }^{1}$ Department of Cardiology, State Key Laboratory of Kidney Disease, Chinese PLA General Hospital, Beijing 100853, China; \\ ${ }^{2}$ Department of Internal Medicine, Beijing Changping Hospital, Beijing 102200, China; ${ }^{3}$ Center for Cardiovascular Research and \\ Alternative Medicine, University of Wyoming College of Health Sciences, Laramie, WY 82071, USA
}

\begin{abstract}
Aldehyde dehydrogenase $2(\mathrm{ALDH} 2)$ is a key mitochondrial enzyme in the metabolism of aldehydes and may have beneficial cardiovascular effects for conditions such as cardiac hypertrophy, heart failure, myocardial I/R injury, reperfusion, arrhythmia, coronary heart disease and atherosclerosis. In this study we investigated the role of ALDH2 in the progression of atherosclerosis and the underlying mechanisms, with a focus on endoplasmic reticulum (ER) stress. A clinical study was performed in 248 patients with coronary heart disease. The patients were divided into two groups according to their ALDH2 genotype. Baseline clinical characteristics and coronary angiography were recorded, and the coronary artery Gensini score was calculated. Serum levels of 4-hydroxy-2-nonenal (4-HNE) were detected. The clinical study revealed that the mutant ALDH2 genotype was an independent risk factor for coronary heart disease. ALDH2 gene polymorphism is closely associated with atherosclerosis and the severity of coronary artery stenosis. Serum levels of 4-HNE were significantly higher in patients with the mutant ALDH2 genotype than in patients with the wild-type ALDH2 genotype. As an in vitro model of atherosclerosis, rat smooth muscle cells (SMCs) were treated with oxygenized low-density lipoprotein (ox-LDL), which significantly elevated the levels of ER markers glucose-regulated protein78 (GRP78), protein kinase R-like ER kinase (PERK), phosphorylated eukaryotic translation initiation factor $\alpha$ subunit ( $p$-elF2 $\alpha$ ), activating transcription factor-4 (ATF-4), CEBP homologous protein (CHOP) and 4-HNE in the cells. All the ox-LDL-induced responses were significantly attenuated in the presence of Alda-1 (an ALDH2 activating agent), and accentuated in the presence of daidzin (an ALDH2 inhibitor). Furthermore, pretreatment with ALDH2 activator Alda-1 significantly decreased ox-LDL-induced apoptosis. Similarly, overexpression of ALDH2 protected SMCs against ox-LDL-induced ER stress as well as ER stress-induced apoptosis. These findings suggest that ALDH2 may slow the progression of atherosclerosis via the attenuation of ER stress and apoptosis in smooth muscle cells.
\end{abstract}

Keywords: atherosclerosis; aldehyde dehydrogenase 2; Alda-1; daidzin; endoplasmic reticulum stress; apoptosis; smooth muscle cells

Acta Pharmacologica Sinica (2018) 39: 48-58; doi: 10.1038/aps.2017.81; published online 31 Aug 2017

\section{Introduction}

Coronary heart disease is one of the leading contributors to the ever-rising morbidity and mortality of cardiovascular disease. Atherosclerosis is considered the predominant pathological basis of coronary heart disease. A wide variety of factors have been identified to contribute to the development of atherosclerosis, including the following: injury to endothelial cells ${ }^{[1]}$,

\footnotetext{
*To whom correspondence should be addressed.

E-mail fengcao8828@163.com (Feng CAO);

Xuyong301@163.com (Yong XU);

jren@uwyo.edu (Jun REN)

Received 2016-12-17 Accepted 2017-05-19
}

abnormal proliferation, migration and phenotypic differentiation of vascular smooth muscle cells (SMCs) ${ }^{[2]}$; and inflammation $^{[3]}$. Recently, ample evidence has been revealed of a pivotal role for endoplasmic reticulum (ER) stress in the pathogenesis of atherosclerosis ${ }^{[4-6]}$. ER stress markers, such as the ER chaperone glucose-regulated protein 78 (GRP78), have been shown to be at elevated levels in advanced atherosclerotic plaques ${ }^{[7]}$. In addition, the unfolded protein response (UPR), which is initiated by ER stress, may play a protective role during the early stage of atherosclerosis. However, with persistent ER stress resulting from chronic exposure to pathological stimuli, large amounts of unfolded proteins can result from ER stress and lead to apoptosis of macrophages and smooth muscle cells ${ }^{[8]}$. 
Aldehyde dehydrogenase 2 (ALDH2) is encoded by the ALDH2 gene, and there are three genotypes: namely, GLU504GLU (GG, wild-type), GLU504LYS (GA, heterozygote) and LYS504LYS (AA, mutant homozygote). ALDH2 is a key mitochondrial enzyme in the metabolism of aldehydes and may have beneficial cardiovascular effects ${ }^{[9,10]}$ for conditions such as cardiac hypertrophy, heart failure, myocardial I/R injury, reperfusion, arrhythmia, coronary heart disease and atherosclerosis. Given the role of ALDH2 in removing toxic aldehydes $^{[11]}$, this study was designed to examine the impact of ALDH2 on the progression of atherosclerosis and the underlying mechanism of action, with a focus on ER stress.

\section{Materials and methods Study design}

A total of 248 patients with coronary heart disease were recruited following clinical diagnosis by cardioangiography. We excluded patients with certain diseases, including those with severe liver and kidney dysfunction (ALT was more than 3 times the upper normal limit and eGFR $<30 \mathrm{~mL} / \mathrm{min}$, respectively), advanced tumors, activated autoimmune disease, acute infectious disease, familial hypercholesterolemia and diabetes. Blood samples were collected for ALDH2 genotyping in a blinded manner. The severity of coronary stenosis was determined by calculating the Gensini score. Additionally, blood homocysteine (Hcy) and high-sensitivity C-reactive protein (hs-CRP) levels were measured. For in vitro study, SMCs were incubated with oxygenized low-density lipoprotein (oxLDL) to simulate atherosclerosis in the presence or absence of factors regulating the enzymatic activity or gene expression of ALDH2. Apoptosis was evaluated by flow cytometry and TUNEL staining. Protein expression levels of ERS markers were determined by Western blotting and immunofluorescence.

\section{Human subjects}

A total of 248 patients with coronary heart disease, as verified by coronary angiography, were recruited and tested for the ALDH2 genotype using the ALDH2 (Glu 504 Lys) Genotyping Kit (BaiO, Shanghai Baiao Technology Corporation, China). All subjects were approved by the Ethics Committee (S2015041-02) to participate in the study. The candidates were divided into an ALDH2-wt group $(n=166)$ and an ALDH2-m group $(n=83)$. Baseline data for the two groups were collected. Coronary angiography was recorded, and the coronary artery Gensini score was calculated. The following standards for the Gensini score were based on the percentage of arterial occlusion: 1 for $1 \%$ to $25 \%, 2$ for $26 \%$ to $50 \%, 4$ for $51 \%$ to $75 \%, 8$ for $76 \%$ to $90 \%$, 16 for $91 \%$ to $99 \%$, and 32 for total occlusion. This score was then multiplied by a weighting for the lesion position in the coronary arterial tree as follows: 5 for the LM (left main artery), 2.5 for the proximal and medial LAD (left anterior descending artery), proximal LCX (left circumflex artery), and 1 for the RCA (right coronary artery) and the remaining vessels. The severity of disease was expressed as the sum of the scores for individual lesions ${ }^{[12]}$. Blood levels of 4-hydroxy-2-nonenal (4-HNE) were detected using an ELISA (R\&B, USA).

\section{Cell culture}

The SMCs were dissociated from the aorta of Sprague-Dawley rats. Endothelial and perithelial cells were removed using a sterile cotton bud to scrape the cells after opening the media longitudinally. The aortic tissue was cut into small pieces (approximately 1-2 $\mathrm{mm}^{2}$ ) using ophthalmic scissors. The pieces were placed on 25- $\mathrm{cm}^{2}$ culture flasks (Corning, USA) with the luminal side down and cultured in Dulbecco's modified Eagle's medium (Gibco, USA) containing 20\% fetal bovine serum in a humidified atmosphere ${ }^{[13]}$.

\section{In vitro treatment regimen}

The SMCs were treated with Alda-1 (20 $\mu \mathrm{mol} / \mathrm{L}$, ALDH2 activating agent, Sigma-Aldrich, USA $)^{[14,15]}$, daidzin $(120 \mu \mathrm{mol} /$ L, ALDH2 inhibitor, Sigma-Aldrich, USA $)^{[16]}$ or a control vehicle, as indicated below, for $48 \mathrm{~h}$. The drugs, which were considered intervention agents, were dissolved in DMSO. To imitate atherosclerosis, the SMCs were treated with ox-LDL $(150 \mu \mathrm{g} / \mathrm{mL})$ in vitro for $24 \mathrm{~h}$. An adenoviral vector encoding ALDH2, including the overexpression and silencing of this, was also applied as an intervention agent.

\section{Measurement of ER stress}

The expression of ER stress-related proteins (glucose-regulated protein78 (GRP78), protein kinase R-like ER kinase (PERK), phosphorylated eukaryotic translation initiation factor a-subunit (p-eIF2 $\alpha$ ), activating transcription factor-4 (ATF-4), CEBP homologous protein (CHOP), and 4-HNE were analyzed using Western blotting and immunofluorescence as markers of ERS. Western blotting was performed as described in detail in the Online Data Supplement. All antibodies were purchased from Abcam (GRP78: ab108613, 1:10000; PERK: 3192, 1:1000; p-eIF2a (Ser51): 9721, 1:1000; ATF-4: ab23760, 5 $\mu \mathrm{g} / \mathrm{mL}$; CHOP: ab10444, 1:250; and 4-HNE: ab46545, 1:1000). Blots were assessed using ImageJ software, and the results are displayed as the ratios of the target protein to GAPDH. The immunofluorescent signal was detected using a confocal laser scanning microscope (Olympus, Fluoview1000, Japan).

\section{Measurement of apoptosis and viability}

Apoptosis was evaluated using flow cytometry and TUNEL staining. SMCs cultured on 6-well plates were digested with pancreatic enzymes after drug intervention, and the cells were washed with PBS three times. Flow cytometry was performed on a FACS Calibur (BD) using the Annexin V-FITC apoptosis detection kit (BD, USA). Annexin V staining was used for early apoptotic cells, and PI staining was used for late apoptosis. The data were analyzed using the FAC Station (BD, USA). The apoptotic cells, labeled in green, were examined using a cell death detection kit (TUNEL staining kit, Roche, Switzerland). The cell nucleus was stained with DAPI (blue). Cell Counting Kit-8 was used to evaluate the viability of the SMCs using a microplate reader (BioTek, USA). 


\section{Statistical analysis}

All analyses were performed using SPSS 16.0 software (SPSS Inc, Chicago, IL, USA). Quantitative data are expressed as the mean \pm SEM. The enumeration data are represented as percentages. If the data were normally distributed and had homogeneity of variance, an unpaired Student's $t$-test was used to calculate significant differences between two groups. A one-way analysis of variance (ANOVA) was used to analyze significant differences among 3 more groups. Otherwise, we used the Wilcoxon rank sum test to analyze the data. Logistic regression analysis was used to assess concordance. A $P$-value of $<0.05$ was considered statistically significant.

\section{Results}

The correlation of ALDH2 polymorphism with the severity of coronary heart disease

To determine the correlation between ALDH2 polymorphism and coronary heart disease, a total of 248 patients who had been diagnosed with coronary heart disease using coronary angiography were recruited of these patients, 84 patients carried the ALDH2 mutation (ALDH2-m), and 164 patients carried wild-type ALDH2 (ALDH2-wt). The patients' anthropometric characteristics are provided in Table 1 . Patients in the ALDH2-m group had more coronary stents than patients in the wild-type group $(1.70 \pm 1.46$ vs $0.74 \pm 1.04$; $P=0.001$; Figure 1A). The Gensini score for the ALDH2-wt group was lower than that for the ALDH2-m group (22.53 \pm 23.23 vs 31.56 \pm 30.41 ; $P=0.026$; Figure 1B). Logistic regression indicated that ALDH2-m $(\mathrm{OR}=2.30, P=0.045$; Figure $1 \mathrm{C})$ was an independent risk factor for coronary heart disease. We also investigated ALDH2 in hypertension-associated coronary artery disease. The results indicated that patients in the ALDH2-m group had more coronary stents than patients in the wild-type group (1.69 \pm 1.82 vs $0.99 \pm 1.32 ; P=0.012$; Table 2$)$. The Gensini score for the ALDH2-wt group was lower than that for the ALDH2m group (28.93 \pm 31.32 vs 40.36 $\pm 36.66 ; P=0.042$; Table 2). Blood

Table 1. Anthropometric data in patients enrolled in the study.

\begin{tabular}{|c|c|c|c|}
\hline Characteristics & ALDH2 wild type $(n=164)$ & ALDH2 mutant type $(n=84)$ & $P$ value \\
\hline Age (year) & $61.61 \pm 13.11$ & $61.36 \pm 13.58$ & 0.887 \\
\hline Male (\%) & $116(70.7)$ & $67(79.8)$ & 0.116 \\
\hline Hypertension (\%) & $111(67.7)$ & $46(54.8)$ & 0.371 \\
\hline Hyperlipidemia* (\%) & $53(32.3)$ & $25(29.8)$ & 0.772 \\
\hline Smoking (\%) & $77(47.0)$ & $41(48.8)$ & 0.654 \\
\hline Alcohol intake (\%) & $43(26.2)$ & $6(7.1)$ & 0.000 \\
\hline BMI (Body mass index, kg/m²) & $25.72 \pm 3.55$ & $26.02 \pm 3.7$ & 0.533 \\
\hline TC (Total cholesterol, mmol/L) & $3.92 \pm 0.91$ & $3.86 \pm 0.87$ & 0.636 \\
\hline TG (Triglyceride, mmol/L) & $1.56 \pm 0.80$ & $1.44 \pm 0.60$ & 0.303 \\
\hline HDL (High-density lipoprotein, mmol/L) & $1.12 \pm 0.27$ & $1.16 \pm 0.36$ & 0.408 \\
\hline LDL (Low-density lipoprotein, mmol/L) & $2.42 \pm 0.78$ & $2.32 \pm 0.79$ & 0.351 \\
\hline APO-A1 (Apolipoprotein-A1, g/L) & $1.35 \pm 0.25$ & $1.36 \pm 0.28$ & 0.561 \\
\hline APO-B (Apolipoprotein-B, g/L) & $0.83 \pm 0.19$ & $0.84 \pm 0.27$ & 0.862 \\
\hline ALT (Alanine aminotransferase, U/L) & $27.03 \pm 34.21$ & $26.39 \pm 19.28$ & 0.799 \\
\hline AST (Aspartate aminotransferase, U/L) & $39.56 \pm 88.11$ & $29.39 \pm 49.55$ & 0.479 \\
\hline BUN (Blood urea nitrogen, mmol/L) & $5.25 \pm 2.03$ & $5.43 \pm 7.77$ & 0.150 \\
\hline CR (Creatinine, $\mu \mathrm{mol} / \mathrm{L})$ & $80.94 \pm 26.56$ & $82.49 \pm 19.92$ & 0.085 \\
\hline Ccr (Creatinine clearance rate, $\mathrm{mL} / \mathrm{min}$ ) & $88.48 \pm 21.32$ & $86.55 \pm 20.87$ & 0.495 \\
\hline
\end{tabular}

*Hyperlipidemia is defined as total cholesterol $>5.17 \mathrm{mmol} / \mathrm{L}(200 \mathrm{mg} / \mathrm{dL})$ or triacylglycerol $>2.3 \mathrm{mmol} / \mathrm{L}(200 \mathrm{mg} / \mathrm{dL})$.

Table 2. Data in patients with hypertension.

\begin{tabular}{|c|c|c|c|}
\hline Characteristics & ALDH2 wild type $(n=111)$ & ALDH2 mutant type $(n=46)$ & $P$ value \\
\hline TC (Total cholesterol, mmol/L) & $3.90 \pm 0.9$ & $3.88 \pm 0.86$ & 0.914 \\
\hline TG (Triglyceride, mmol/L) & $1.6 \pm 0.82$ & $1.37 \pm 0.5$ & 0.163 \\
\hline HDL (High-density lipoprotein, mmol/L) & $1.11 \pm 0.26$ & $1.22 \pm 0.35$ & 0.135 \\
\hline LDL (Low-density lipoprotein, mmol/L) & $2.43 \pm 0.78$ & $2.30 \pm 0.81$ & 0.341 \\
\hline APO-A1 (Apolipoprotein-A1, g/L) & $1.35 \pm 0.24$ & $1.37 \pm 0.27$ & 0.518 \\
\hline APO-B (Apolipoprotein-B, g/L) & $0.82 \pm 0.19$ & $0.84 \pm 0.33$ & 0.654 \\
\hline Total number of stents & $0.99 \pm 1.32$ & $1.69 \pm 1.82$ & 0.012 \\
\hline Gensini score & $28.93 \pm 31.32$ & $40.36 \pm 36.66$ & 0.042 \\
\hline
\end{tabular}


A
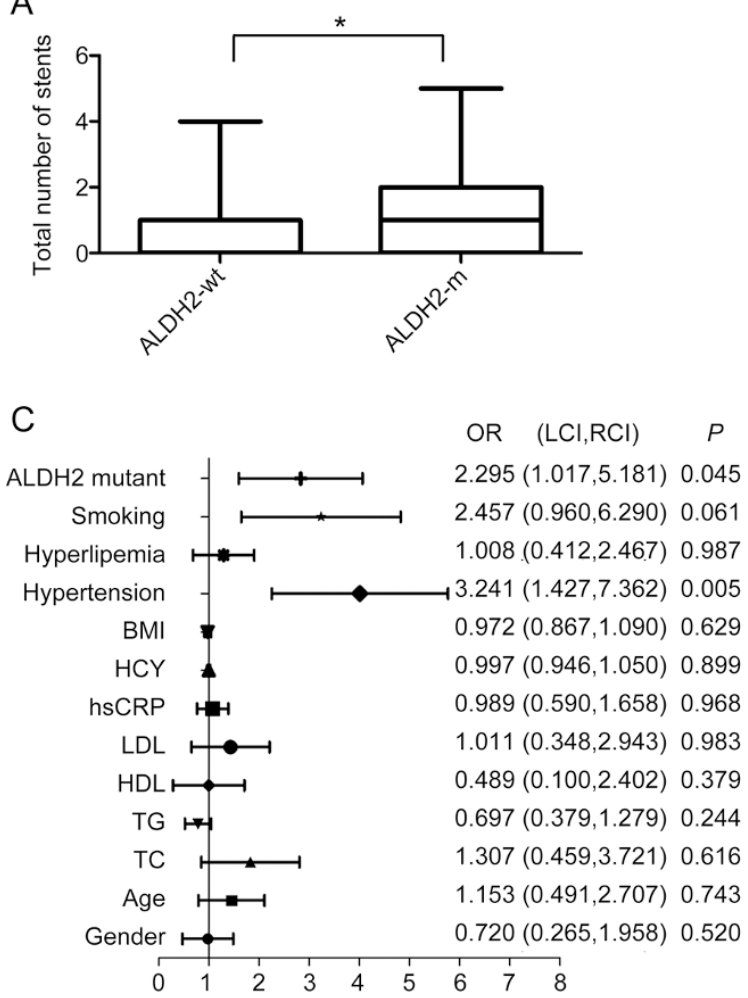

B

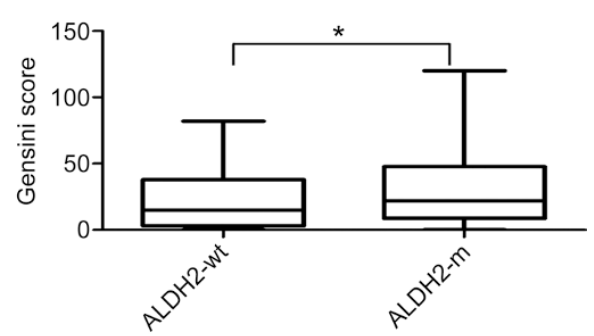

$\mathrm{D}$

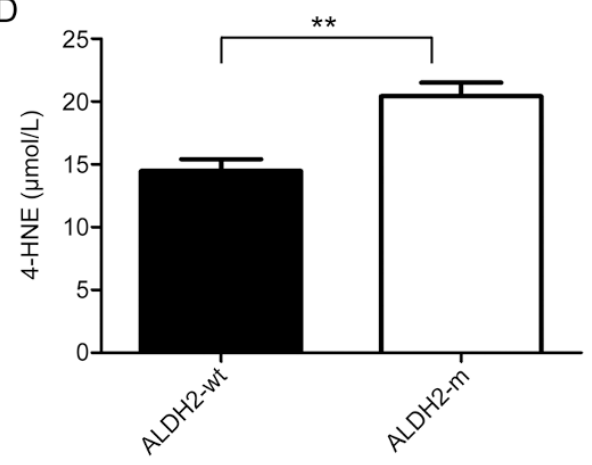

Figure 1. The analysis of ALDH2 polymorphism in humans reveals that the ALDH2 mutation correlates with atherosclerosis associated with elevated levels of 4-HNE. (A) The total number of coronary artery stents in patients with wild-type ALDH2 ( $n=164)$ and with the ALDH2 mutation (including homozygous and heterozygous mutations, $n=84, P=0.01$ ) is shown. The genotyping of ALDH2 was determined in human blood; (B) Gensini scores in patients with wild-type ALDH2 and the ALDH2 mutation $(P=0.026)$; (C) Logistic regression indicates the ALDH2 mutation $(\mathrm{OR}=2.30, P=0.045)$ as an independent risk factor for coronary heart disease; and (D) Serum 4-HNE levels in wild-type ALDH2 and ALDH-mutant groups are shown ( ${ }^{*} P<0.05$; $\left.{ }^{* *} P<0.01\right)$.

samples were collected from 44 ALDH2-wt patients and 44 ALDH2-m patients. The ELISA revealed higher 4-HNE levels in the ALDH2-m group than in the ALDH2-wt group (20.43 \pm 5.87 vs $14.47 \pm 5.16 \mu \mathrm{mol} / \mathrm{L} ; P<0.01$; Figure 1D).

Activation of ALDH2 inhibits ERS in the progression of atherosclerosis ER stress has been shown to play an important role in the pathogenesis of atherosclerosis ${ }^{[17]}$. To evaluate the role of ALDH2 in atherosclerosis, primary smooth muscle cells isolated from the rat aorta were treated with ox-LDL to mimic atherosclerosis in vitro in the presence and absence of an ALDH2 activator (Alda-1) or an ALDH2 inhibitor (daidzin). Western blot and immunofluorescence findings indicated that ox-LDL significantly elevated levels of the ER chaperone GRP78, the effect of which was accentuated by daidzin $(P<0.01$, Figure $2 C)$ and attenuated by Alda- $1(P<0.01$, Figure 2C). The PERK, p-eIF2a, ATF-4 and 4-HNE levels displayed similar results to those for GRP78 (Figure 2B, 2D, 2E, 2F).

\section{The effect of ALDH2 activation on ER stress-induced apoptosis}

In the advanced stage of ER stress, apoptotic signaling molecules such as CHOP may contribute to cell apoptosis ${ }^{[18-21]}$. TUNEL analysis revealed that pretreatment with the ALDH2 activator Alda-1 reduced ox-LDL-mediated cell apoptosis, which was in contrast to the effect of pretreatment with daidzin $(0.21 \pm 0.02$ vs $0.42 \pm 0.10$; $P<0.001$; Figure $3 A, 3 B)$. Similar results were obtained with flow cytometric analysis of Annexin V and propidium iodide (PI) staining (Figure 3C, 3D). The increase in the protein level of CHOP in cultured SMCs that were exposed to ox-LDL was abolished by Alda-1 and enhanced by daidzin (Figure 3E, 3F). Cell numbers and viability levels were significantly reduced by daidzin compared to

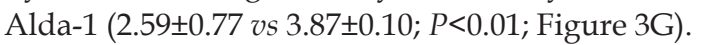

\section{Overexpression of ALDH2 protects SMCs against ER stress}

It has been shown that ALDH2 activation can protect smooth muscle cells from ER stress injury. In an effort to understand the mechanism underlying ALDH2-mediated protection, primary SMCs were transfected with an adenoviral vector encoding ALDH2 or shRNA-ALDH2 (AD-ALDH2 and ADshRNA-ALDH2). Control VSMCs were transfected with a corresponding recombinant adenoviral vector (rAD). Levels of ALDH2 were detected using RT-PCR and Western blotting. Our data revealed that AD-ALDH2 transfection at a multiplicity of infection (MOI) of 200 may achieve a higher efficiency than AD-ALDH2 transfection at an MOI of 50 or 100 , albeit with a higher rate of cell death. The RNA levels were significantly higher at an MOI of 100 than at an MOI of 
A



Smooth muscle cells

identification

(Green: a-SMA, blue: DAPI)

D
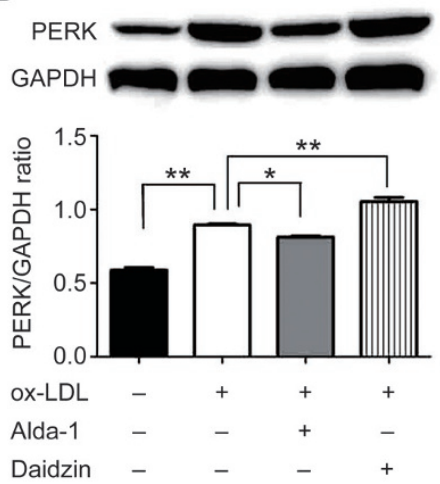

G
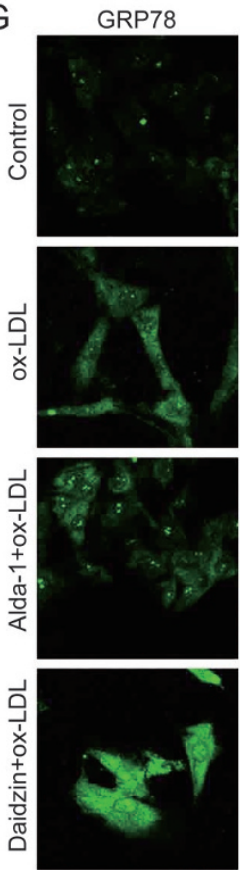

B
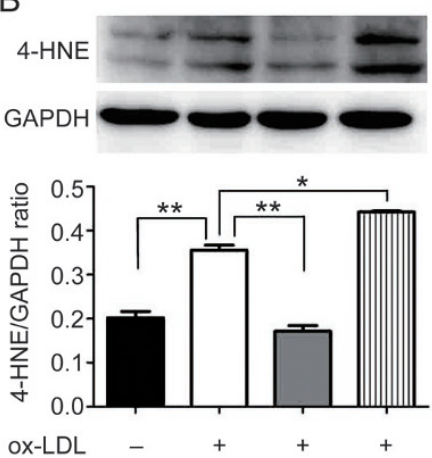

Alda-1

Daidzin

\section{$E$}


C


ox-LDL - $\quad+\quad+\quad+$

Alda-1 - $\quad-\quad+\quad+$

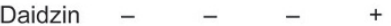

F
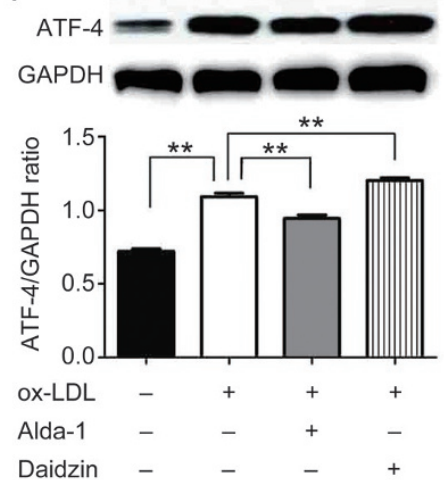

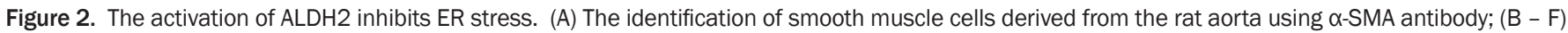

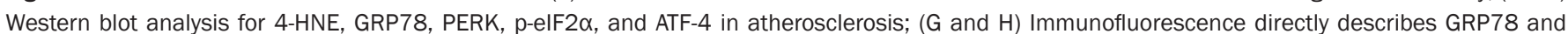
ATF-4 ( $\left.{ }^{*} P<0.05,{ }^{* *} P<0.01\right)$.

$50(9.47 \pm 2.72$ vs $2.95 \pm 1.24 ; P<0.01$; Figure $4 \mathrm{~A})$. The levels of GRP78, PERK, p-eIF2a, ATF-4, and 4-HNE were elevated fol- lowing shALDH2 infection, which indicated overt ER stress. However, this increase in the levels of these ER stress proteins 
A
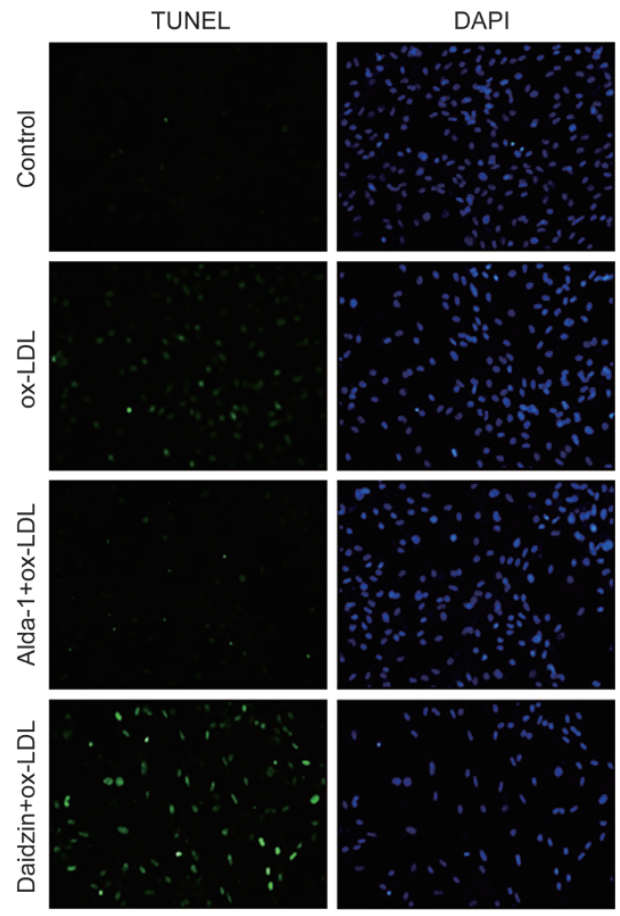

C
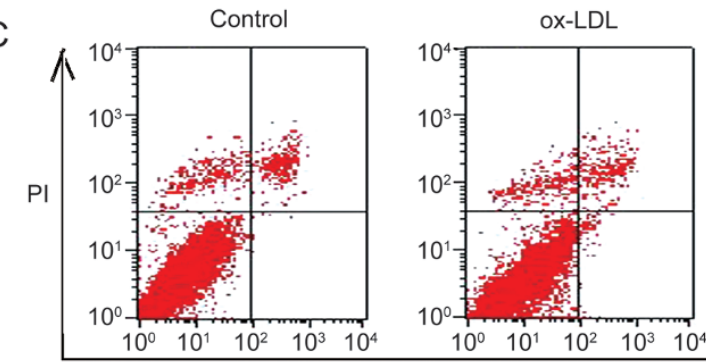

B
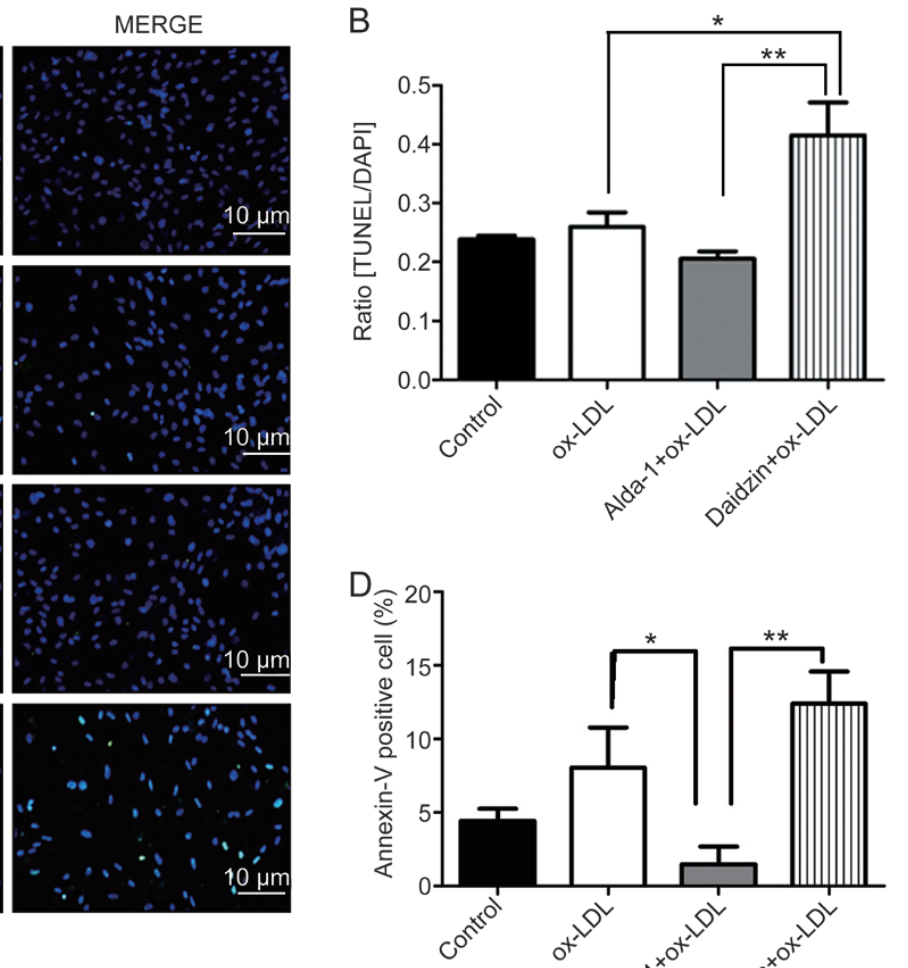

E

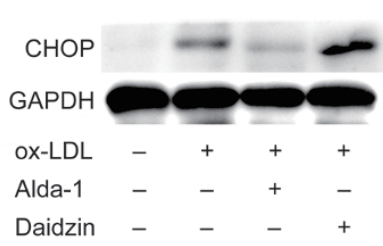

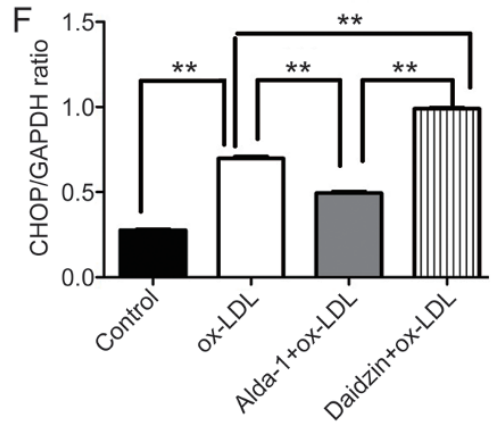

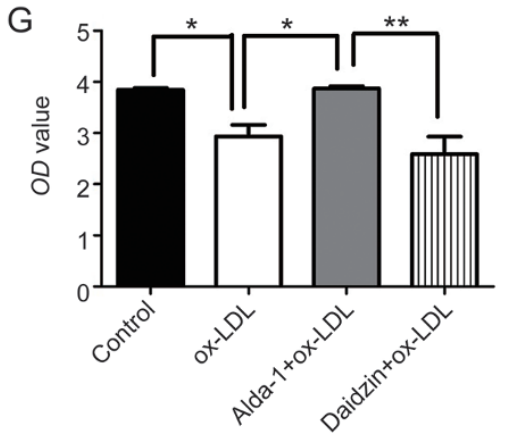

Figure 3. The effect of ALDH2 activation on ER stress-elicited apoptosis. (A-D) ER stress-induced apoptosis was assessed using TUNEL staining and flow cytometric analysis in response to the ALDH2 activator Alda-1 and the ALDH2 inhibitor daidzin; (E and F) Western blot analysis shows the role of ER stress in the ALDH2-induced apoptotic response; $(G)$ The effect of Alda-1 and daidzin on the viability and number of SMC cells.

was attenuated by AD-ALDH2 (Figure 4B, 4C, 4D, 4E, and 4F).

\section{ALDH2 overexpression attenuates ER stress-induced apoptosis}

Flow cytometric analysis was employed to examine whether ALDH2 overexpression or knockdown directly regulated apoptosis. Flow cytometric analysis revealed that AD-ALDH2 attenuated ox-LDL-induced cell death and that shALDH2 exacerbated ox-LDL-induced cell death (Figure 5A and 5B). Likewise, the rise in $\mathrm{CHOP}$ protein expression that followed an ox-LDL challenge was abolished in the presence of AD- 


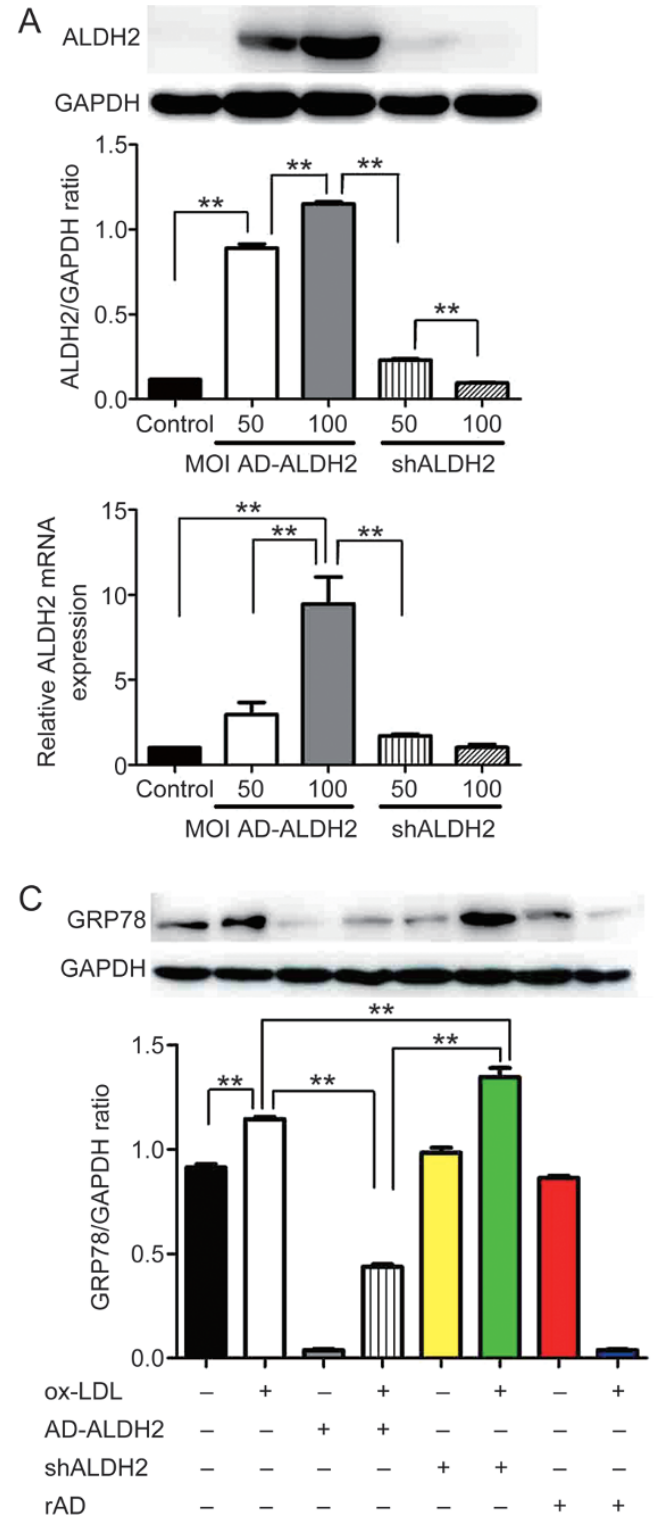

$E$
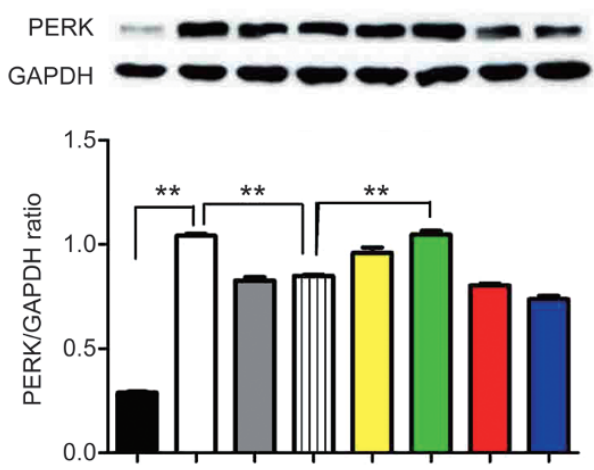

ox-LDL

AD-ALDH2

ShALDH2

rAD
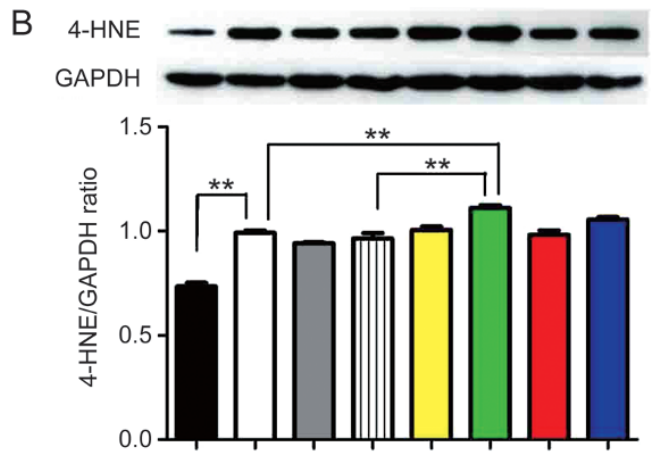

ox-LDL

AD-ALDH2 $-(-1+2+$

shALDH2

rAD


F


ox-LDL

AD-ALDH2

shALDH2

rAD

Figure 4A-4F. Transfection with ALDH2 protects SMCs against ER stress. (A) The optimal MOI is determined by Western blotting and real-time PCR; (B-F) Western blot analysis for 4-HNE, GRP78, p-elF2 $\alpha$, PERK, and ATF-4. ${ }^{*} P<0.05 ;{ }^{* *} P<0.01$. 
G

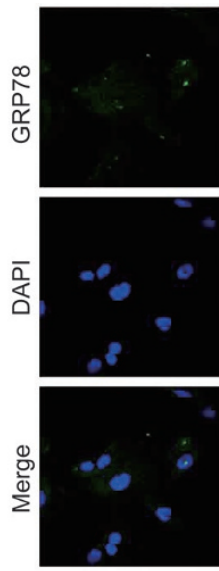

Control

$\mathrm{H}$
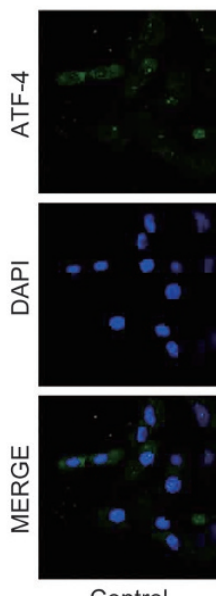

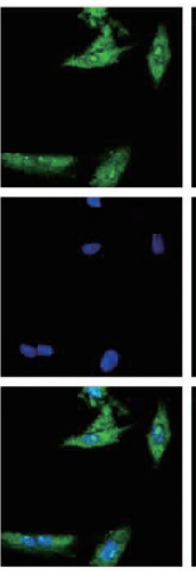

ox-LDL
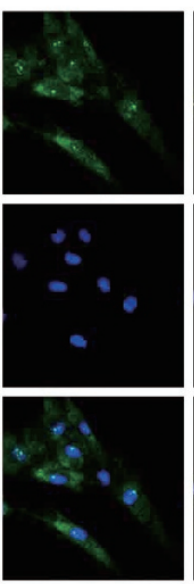

ox-LDL
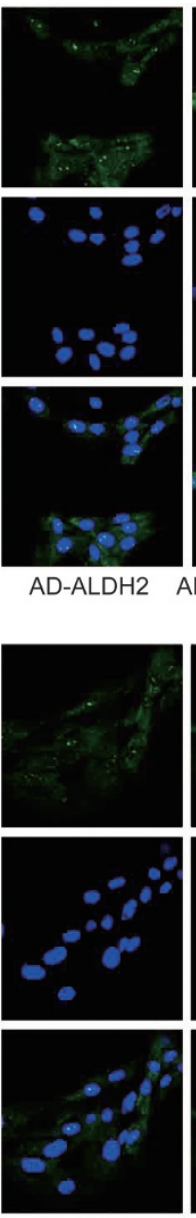

AD-ALDH2
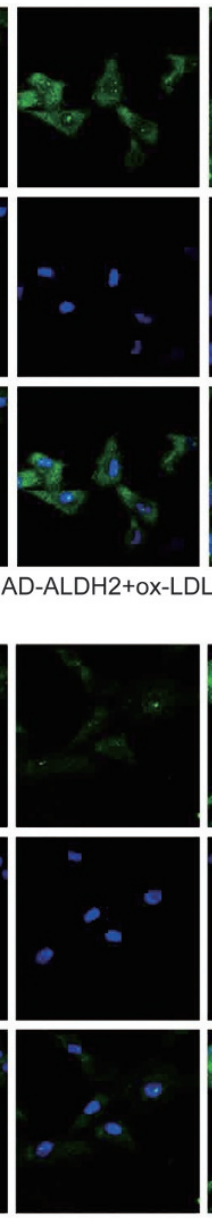

AD-ALDH2+ox-LD
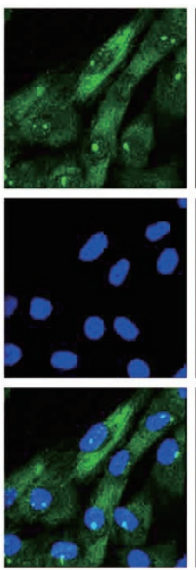

sh-ALDH2
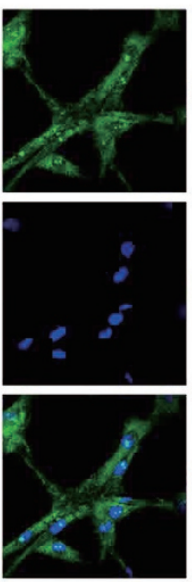

sh-ALDH2
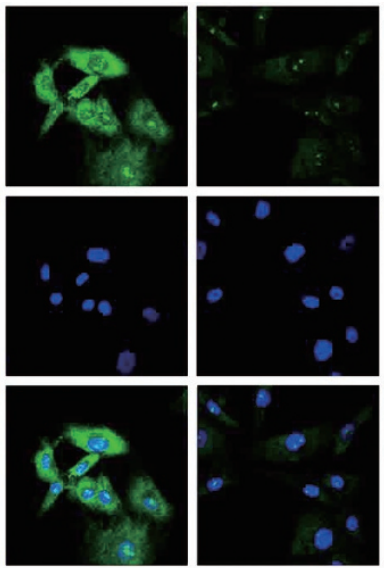

rAD
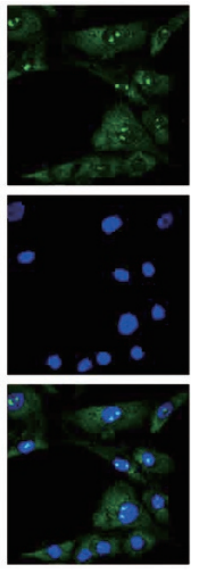

rAD+ox-LDL
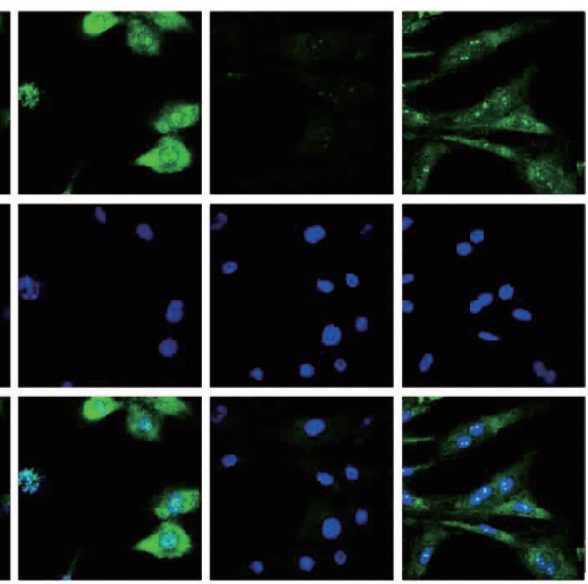

rAD

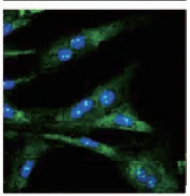

rAD+ox-LDL

Figure 4G, 4H. (G and H) Immunofluorescence for GRP78 and ATF-4 after transfection of AD-ALDH2 or sh-ALDH2 into SMCs.

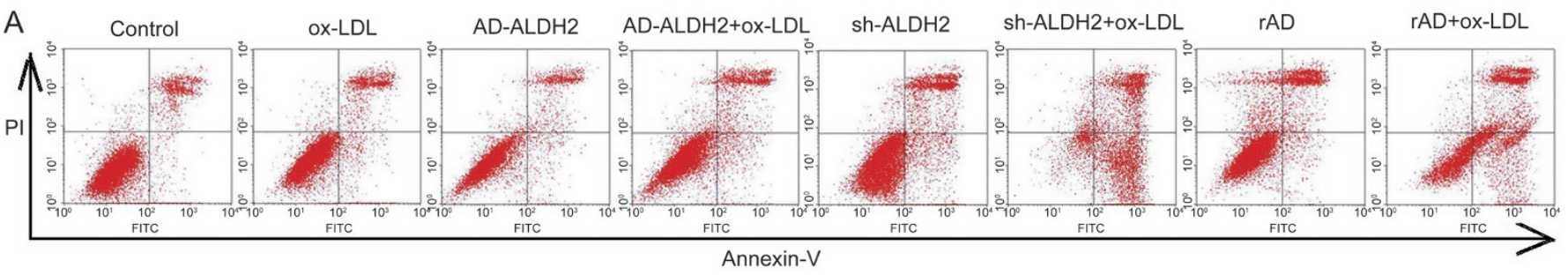

$\mathrm{B}$

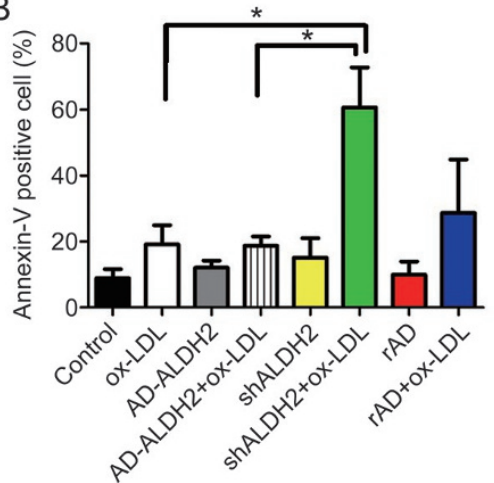

C

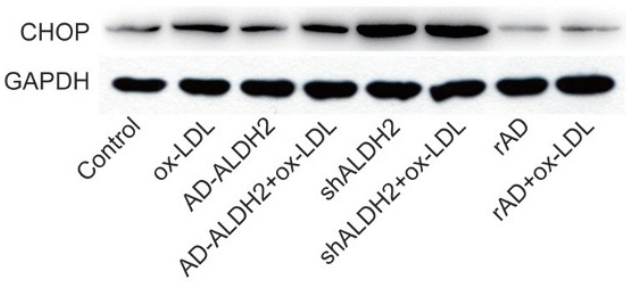

D

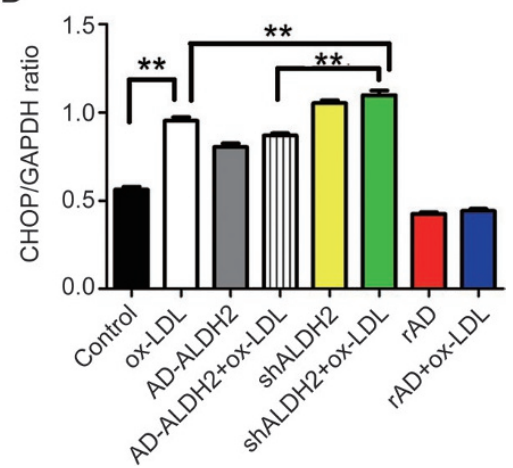

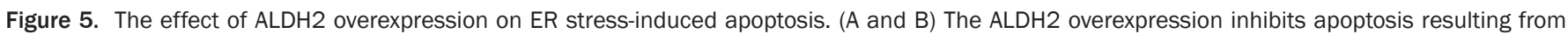


quantification shows that ALDH2 inhibited apoptosis via suppression of ER stress. ${ }^{* *} P<0.01$. 


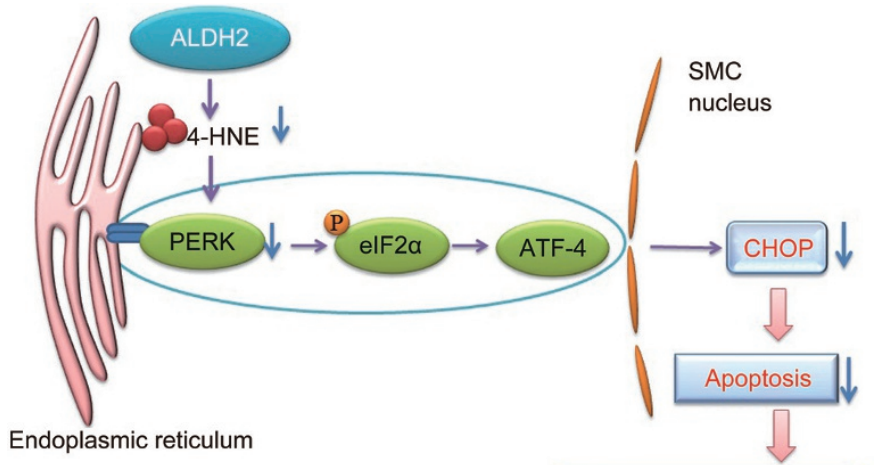

Plaque progression

Figure 6. The effect of ALDH2 on 4-HNE-induced ER stress is shown. In the early stages of ER stress, the UPR is induced and maintains ER homeostasis via PERK. PERK phosphorylates elF2 $\alpha$, which leads to expression of the gene encoding the transcription factor ATF-4. In the advanced stage of ER stress, the downstream target CHOP can trigger apoptosis, which causes the progression of plaques. ALDH2 is capable of attenuating ER stress and apoptosis in smooth muscle cells by detoxifying 4-HNE.

ALDH2 and accentuated in the presence of AD-shALDH2 (Figure 5C, 5D).

\section{Discussion}

Coronary heart disease is associated with complex traits that result from various etiological cues ${ }^{[22]}$, including smoking, obesity and hyperlipidemia ${ }^{[23,24]}$. Intense efforts have been made in the field of ischemic cardiomyopathy to develop various genomic and proteomic techniques ${ }^{[25]}$. Many studies have shown that the ALDH2 rs671 polymorphism may be associated with coronary heart disease ${ }^{[26-29]}$. Takagi and colleagues suggested that the ALDH2 mutation may serve as a risk factor for coronary heart disease during early life ${ }^{[30]}$; however, the opposite findings have also been reported. Narita et al found that patients without an ALDH2 mutation had less severe carotid atherosclerosis $^{[31]}$. Our clinical findings showed that the ALDH2 gene mutation is an independent risk factor for coronary heart disease, which may possibly be due to the ALDH2 mutant having less capacity to remove aldehydes; however, the precise mechanism remains elusive. The in vitro experiments revealed that ALDH2 inhibited the progression of atherosclerosis via attenuating ER stress and apoptosis in smooth muscle cells.

A total of 248 patients with coronary heart disease were recruited for our current study with the aim of discerning the relationship between ALDH2 polymorphism and coronary heart disease. Of these patients, 164 had the wild-type ALDH2 phenotype (66.1\%), and the remaining 33.9\% had the ALDH2 mutation, consistent with Hardy-Weinberg equilibrium. In addition, the majority of patients enrolled were male patients. Compared to women, men were found to have a higher prevalence of coronary artery disease ${ }^{[32]}$.

Our findings show the pivotal role of the ALDH2 mutation in the incidence of coronary heart disease. Aldehydes, includ- ing 4-HNE, are commonly found in ischemic, hypertrophic, and failing hearts and atherosclerotic lesions ${ }^{[33]}$. ALDH2 plays a critical role in clearing endogenous aldehydes such as 4-HNE during the pathogenesis of coronary heart disease. This result is consistent with reports from Lishun Wang ${ }^{[34]}$ and Elizabeth Murphy ${ }^{[35]}$. Findings from a meta-analysis by Zhang and colleagues ${ }^{[36]}$ suggested that variant A allele carriers displayed a $48 \%$ higher risk of coronary artery disease (CAD) than individuals who were homozygous for the GG allele $(\mathrm{OR}=1.48)$. The risk of CAD among those with the mutated gene was 1.58-fold greater than that among those with wildtype alleles. The facilitation of acetaldehyde metabolism by ALDH2 overexpression may prevent acetaldehyde-induced cell injury and activation of stress signals ${ }^{[37]}$. ALDH2 may ablate alcohol-induced changes in association with alleviating ER stress (IRE1a, eIF2a, GRP78 and CHOP), which suggests the involvement of ER stress in alcohol-induced myocardial injury ${ }^{[38]}$. The findings from our present work suggest that ALDH2-mutant may be an independent risk factor for coronary heart disease independent of alcoholism because patients with wild-type ALDH2 drank more alcohol than patients with the ALDH2 mutant. As a result, in vitro experiments were performed to investigate the mechanism.

In our study, ox-LDL was used as a classic factor to mimic atherosclerosis in vitro. Our data revealed that ox-LDL promoted ER stress, as evidenced by upregulation of the ER stress markers GRP78, PERK and p-eIF2a ER, which indicated a role for ER stress in the onset and development of atherosclerosis. These results were in accordance with a previous study by Ivanova and colleagues ${ }^{[39]}$. Many physiological and pathological stress factors can induce ER stress, such as ischemia, hypoxia, ox-LDL, aldehydes, angiotensin II, tumor necrosis factor- $\alpha$ and lipid metabolism ${ }^{[40]}$. Methylglyoxal, a toxic aldehydes, may activate ER stress through the three classical unfolded protein sensors in a manner reminiscent of $4-\mathrm{HNE}^{[41,42]}$. In our study, ALDH2 protected cells from aldehyde-mediated damage through effective clearance ${ }^{[43,44]}$. Our data showed elevated 4-HNE levels in the absence of ALDH2, the effect of which was nullified by elevated ALDH2 activity or ALDH2 overexpression.

In the advanced stages of ER stress, p-eIF2a activates expression of the gene encoding the transcription factor ATF- $4^{[45]}$ and its downstream target CHOP, which leads to apoptosis ${ }^{[46,47]}$. As a result, this classical signal cascade of PERK-p-eIF2a-ATF4-CHOP was evaluated for possible interplay between ALDH2 and ER stress. In our study, the activation of ALDH2 using Alda-1 or ALDH2 overexpression downregulated the levels of GRP78, PERK, p-eIF2a, ATF-4, and CHOP protein in smooth muscle cells, whereas the opposite response was seen with the ALDH2 inhibitor daidzin or with ALDH2 knockdown. In the advanced stage of ER stress, the persistent accumulation of misfolded proteins can trigger apoptosis. Using TUNEL and flow cytometry to assess apoptosis, we observed the attenuation of ER stress-induced apoptosis by ALDH2. Apoptosis of the SMCs within the plaque may destabilize the lesion owing to decreased collagen production and consequent thinning of 
the protective fibrous cap in the advanced stage of atheroscle$\operatorname{rosis}^{[48-51]}$.

Although the data from our study bear some clinical relevance, several limitations remain. First, 4-HNE may be detoxified by various factors, such as inflammatory factors, and may not represent all aldehydes. Second, the in vitro cell experiments cannot fully simulate human atherosclerosis; thus, animal studies are warranted to verify the precise mechanism of action underlying the ALDH2-mediated effect on the progression of atherosclerosis.

Taken these findings together, ALDH2 may prevent the progression of atherosclerosis via attenuating ERS and apoptosis in smooth muscle cells. ALDH2 genotyping may be used to screen for risk factors for coronary heart disease. Patients with coronary heart disease who carry the ALDH2 gene mutation may benefit from treatment with an ALDH2 activator.

In conclusion, ALDH2 gene polymorphism is closely associated with atherosclerosis and the severity of stenosis in the coronary arteries. ALDH2 gene mutation may be an independent risk factor for coronary heart disease associated with elevated ER stress. ALDH2 prevent the progression of atherosclerosis via attenuating ER stress and apoptosis in smooth muscle cells. ALDH2 genotypes may serve as a screening focus for coronary heart disease risk, and mitochondrial enzymes may be considered a new therapeutic target for cardiovascular disease.

\section{Acknowledgements}

This work was supported by the National Key Research Program of China (№ 2016YFA0100900, 2016YFC1300301), the National Fund for Distinguished Young Scientists of China (№ 81325009), the National Natural Science Foundation of China (Nos 81227901, 81530058, and 81570272), and the Beijing Natural Science Foundation (№ 7152131).

\section{Author contribution}

Yong XU and Feng CAO designed the research study and wrote the manuscript; Mei-yan YANG conducted the experiments, analyzed the data, and wrote the manuscript; Ya-bin WANG, Bo HAN, Bo YANG, Yu-wei QIANG, Yan ZHANG, Zhao WANG, Xu HUANG, Jie LIU, Yun-dai CHEN conducted some of the experiments; and Jun REN participated in discussions and wrote the manuscript.

\section{References}

1 Chistiakov DA, Orekhov AN, Bobryshev YV. Endothelial barrier and its abnormalities in cardiovascular disease. Frontiers Physiol 2015; 6: $1-11$.

2 Owens GK, Kumar MS, Wamhoff BR. Molecular regulation of vascular smooth muscle cell differentiation in development and disease. Physiol Rev 2004; 84: 767-801.

3 Libby P. History of discovery: inflammation in atherosclerosis. Arterioscler Thromb Vasc Biol 2012; 32: 2045-51.

4 Tabas I. The role of endoplasmic reticulum stress in the progression of atherosclerosis. Circ Res 2010; 107: 839-50.

5 Glembotski CC. Endoplasmic reticulum stress in the heart. Circ Res 2007; 101: 975-84.
6 Song G, Zong C, Zhang Z, Yu Y, Yao S, Jiao P, et al. Molecular hydrogen stabilizes atherosclerotic plaque in low-density lipoprotein receptor-knockout mice. Free Radic Biol Med 2015; 87: 58-68.

7 Myoishi M, Hao H, Minamino T, Watanabe K, Nishihira K, Hatakeyama $\mathrm{K}$, et al. Increased endoplasmic reticulum stress in atherosclerotic plaques associated with acute coronary syndrome. Circulation 2007; 116: 1226-33.

8 Zhou AX, Tabas I. The UPR in atherosclerosis. Semin Immunopathol 2013; 35: 321-32.

9 Gong D, Zhang H, Hu S. Mitochondrial aldehyde dehydrogenase 2 activation and cardioprotection. J Mol Cell Cardiol 2013; 55: 58-63.

10 Chen CH, Budas GR, Churchill EN, Disatnik MH, Hurley TD, MochlyRosen D. Activation of aldehyde dehydrogenase-2 reduces ischemic damage to the heart. Science 2008; 321: 1493-5.

11 Mali VR, Deshpande M, Pan G, Thandavarayan RA, Palaniyandi SS. Impaired ALDH2 activity decreases the mitochondrial respiration in H9C2 cardiomyocytes. Cell Signal 2016; 28: 1-6.

12 Tousoulis D, Siasos G, Maniatis K, Oikonomou E, Kioufis S, Zaromitidou $\mathrm{M}$, et al. Serum osteoprotegerin and osteopontin levels are associated with arterial stiffness and the presence and severity of coronary artery disease. Int J Cardiol 2013; 167: 1924-8.

13 Cao C, Ji X, Luo X, Zhong L. Gingipains from Porphyromonas gingivalis promote the transformation and proliferation of vascular smooth muscle cell phenotypes. Int J Clin Exp Med 2015; 8: 18327-34.

14 Dolle L, Gao B. Pharmacological chaperone therapies: Can aldehyde dehydrogenase activator make us healthier? J Hepatol 2015; 62: 1228-30.

15 Zhong W, Zhang W, Li Q, Xie G, Sun Q, Sun X, et al. Pharmacological activation of aldehyde dehydrogenase 2 by Alda- 1 reverses alcoholinduced hepatic steatosis and cell death in mice. J Hepatol 2015; 62 : 1375-81.

$16 \mathrm{Xu} \mathrm{B}$, Li P, Zhang G. Comparative pharmacokinetics of puerarin, daidzin, baicalin, glycyrrhizic acid, liquiritin, berberine, palmatine and jateorhizine by liquid chromatography-mass spectrometry after oral administration of Gegenqinlian decoction and active components alignment (ACA) to rats. J Chromatogr B Analyt Technol Biomed Life Sci 2015; 988: 33-44.

17 Mozzini C, Fratta Pasini A, Garbin U, Stranieri C, Pasini A, Vallerio P, et al. Increased endoplasmic reticulum stress and Nrf2 repression in peripheral blood mononuclear cells of patients with stable coronary artery disease. Free Radic Biol Med 2014; 68: 178-85.

18 Wang M, Kaufman RJ. Protein misfolding in the endoplasmic reticulum as a conduit to human disease. Nature 2016; 529: 32635.

19 Zhang Q, Liu J, Chen S, Liu J, Liu L, Liu G, et al. Caspase-12 is involved in stretch-induced apoptosis mediated endoplasmic reticulum stress. Apoptosis 2016; 21: 432-42.

20 Zhang J, Yi M, Zha L, Chen S, Li Z, Li C, et al. Sodium butyrate induces endoplasmic reticulum stress and autophagy in colorectal cells: implications for apoptosis. PLoS One 2016; 11: e0147218.

21 Gu S, Chen C, Jiang X, Zhang Z. ROS-mediated endoplasmic reticulum stress and mitochondrial dysfunction underlie apoptosis induced by resveratrol and arsenic trioxide in A549 cells. Chem Biol Interact 2016; 245: 100-9.

22 Murray CJL, Lopez AD. Global mortality, disability, and the contribution of risk factors: global burden of disease study. Lancet 1997; 349: 1436-42.

23 Toth PP, Davidson MH. High-density lipoproteins: marker of cardiovascular risk and therapeutic target. J Clin Lipidol 2010; 4: 359-64.

24 Tall AR. Cholesterol efflux pathways and other potential mechanisms 
involved in the athero-protective effect of high density lipoproteins. J Intern Med 2008; 263: 256-73.

25 Takeuchi F, Yokota M, Yamamoto K, Nakashima E, Katsuya T, Asano $\mathrm{H}$, et al. Genome-wide association study of coronary artery disease in the Japanese. Eur J Hum Genet 2012; 20: 333-40.

26 Han $\mathrm{H}$, Wang $\mathrm{H}$, Yin Z, Jiang $\mathrm{H}$, Fang M, Han J. Association of genetic polymorphisms in $\mathrm{ADH}$ and $\mathrm{ALDH} 2$ with risk of coronary artery disease and myocardial infarction: a meta-analysis. Gene 2013; 526: 13441.

27 Gu JY, Li LW. ALDH2 Glu504Lys polymorphism and susceptibility to coronary artery disease and myocardial infarction in east Asians: a meta-analysis. Arch Med Res 2014; 45: 76-83.

28 Jia K, Wang H, Dong P. Aldehyde dehydrogenase 2 (ALDH2) Glu504Lys polymorphism is associated with hypertension risk in Asians: a metaanalysis.pdf. Int J Clin Exp Med 2015; 8: 10767-72.

29 Ebert AD, Kodo K, Liang P, Wu H, Huber BC, Riegler J, et al. Characterization of the molecular mechanisms underlying increased ischemic damage in the aldehyde dehydrogenase 2 genetic polymorphism using a human induced pluripotent stem cell model system. Sci Transl Med 2014; 6: 255ra130.

30 Takagi S, Iwai N, Yamauchi R, Kojima S, Yasuno S, Baba T, et al. Aldehyde dehydrogenase 2 gene is a risk factor for myocardial infarction in Japanese men. Hypertens Res 2002; 25: 677-81.

31 Narita M, Kitagawa K, Nagai Y, Hougaku H, Hashimoto H, Sakaguchi $\mathrm{M}$, et al. Effects of aldehyde dehydrogenase genotypes on carotid atherosclerosis. Ultrasound Med Biol 2003; 29: 1415-9.

32 Li TY, Tse MY, Pang SC, McLellan CS, King WD, Johri AM. Sex differences of the natriuretic peptide polymorphism associated with angiographic coronary atherosclerosis. Cardiol Res 2017; 8: 1-6.

33 Hill BG, Bhatnagar A. Beyond reactive oxygen species: aldehydes as arbitrators of alarm and adaptation. Circ Res 2009; 105: 1044-6.

34 Duan Y, Gao Y, Zhang J, Chen Y, Jiang Y, Ji J, et al. Mitochondrial aldehyde dehydrogenase 2 protects gastric mucosa cells against DNA damage caused by oxidative stress. Free Radic Biol Med 2016; 93 : 165-76.

35 Lagranha CJ, Deschamps A, Aponte A, Steenbergen C, Murphy E. Sex differences in the phosphorylation of mitochondrial proteins result in reduced production of reactive oxygen species and cardioprotection in females. Circ Res 2010; 106: 1681-91.

36 Zhang LL, Wang YQ, Fu B, Zhao SL, Kui Y. Aldehyde dehydrogenase 2 (ALDH2) polymorphism gene and coronary artery disease risk: a metaanalysis. Genet Mol Res 2015; 14: 18503-14.

37 Li SY, Gomelsky M, Duan J, Zhang Z, Gomelsky L, Zhang X, et al. Overexpression of aldehyde dehydrogenase-2 (ALDH2) transgene prevents acetaldehyde-induced cell injury in human umbilical vein endothelial cells: role of ERK and p38 mitogen-activated protein kinase. J Biol Chem 2004; 279: 11244-52.

38 Li SY, Gilbert SA, Li Q, Ren J. Aldehyde dehydrogenase-2 (ALDH2) ameliorates chronic alcohol ingestion-induced myocardial insulin resistance and endoplasmic reticulum stress. J Mol Cell Cardiol 2009; 47: 247-55.

39 Ivanova EA, Orekhov AN. The role of endoplasmic reticulum stress and unfolded protein response in atherosclerosis. Int J Mol Sci 2016; 17. pii: E193. doi: 10.3390/ijms17020193.

40 Nickson P, Toth A, Erhardt P. PUMA is critical for neonatal cardiomyocyte apoptosis induced by endoplasmic reticulum stress. Cardiovasc Res 2007; 73: 48-56.

41 Palsamy P, Bidasee KR, Ayaki M, Augusteyn RC, Chan JY, Shinohara T. Methylglyoxal induces endoplasmic reticulum stress and DNA demethylation in the Keap1 promoter of human lens epithelial cells and age-related cataracts. Free Radic Biol Med 2014; 72: 134-48.

42 Chistiakov DA, Sobenin IA, Orekhov AN, Bobryshev YV. Role of endoplasmic reticulum stress in atherosclerosis and diabetic macrovascular complications. Biomed Res Int 2014; 2014: 610140.

43 Guo JM, Liu AJ, Zang P, Dong WZ, Ying L, Wang W, et al. ALDH2 protects against stroke by clearing 4-HNE. Cell Res 2013; 23: 91530.

$44 \mathrm{Ma} \mathrm{H}$, Guo R, Yu L, Zhang Y, Ren J. Aldehyde dehydrogenase 2 (ALDH2) rescues myocardial ischaemia/reperfusion injury: role of autophagy paradox and toxic aldehyde. Eur Heart J 2011; 32: 1025-38.

45 Ron D, Walter P. Signal integration in the endoplasmic reticulum unfolded protein response. Nat Rev Mol Cell Biol 2007; 8: 519-29.

46 Tabas I, Ron D. Integrating the mechanisms of apoptosis induced by endoplasmic reticulum stress. Nat Cell Biol 2011; 13: 184-90.

47 Szegezdi E, Logue SE, Gorman AM, Samali A. Mediators of endoplasmic reticulum stress-induced apoptosis. EMBO Rep 2006; 7: $880-5$.

48 Naghavi M, Libby P, Falk E, Casscells SW, Litovsky S, Rumberger J, et al. From vulnerable plaque to vulnerable patient: a call for new definitions and risk assessment strategies: Part I. Circulation 2003; 108: 1664-72.

49 Naghavi M, Libby P, Falk E, Casscells SW, Litovsky S, Rumberger J, et al. From vulnerable plaque to vulnerable patient: a call for new definitions and risk assessment strategies: Part II. Circulation 2003; 108: 1772-8.

50 Tabas I. Consequences and therapeutic implications of macrophage apoptosis in atherosclerosis: the importance of lesion stage and phagocytic efficiency. Arterioscler Thromb Vasc Biol 2005; 25: 225564.

51 Clarke MC, Figg N, Maguire JJ, Davenport AP, Goddard M, Littlewood TD, et al. Apoptosis of vascular smooth muscle cells induces features of plaque vulnerability in atherosclerosis. Nat Med 2006; 12: 1075-80. 